\title{
Necroptosis: A novel manner of cell death, associated with stroke (Review)
}

\author{
CHENGLIN LIU*, KAI ZHANG ${ }^{*}$, HAITAO SHEN, XIYANG YAO, QING SUN and GANG CHEN \\ Department of Neurosurgery, The First Affiliated Hospital of Soochow University, Suzhou, Jiangsu 215006, P.R. China
}

Received December 29, 2015; Accepted October 24, 2017

DOI: $10.3892 /$ ijmm.2017.3279

\begin{abstract}
Cell death is indispensable in the physiology, pathology, growth, development, senility and death of an organism. In recent years, the identification of a highly regulated form of necrosis, known as necroptosis, has challenged the traditional concept of necrosis and apoptosis, which are two major modes of cell death. This novel manner of cell death is similar in form to necrosis in terms of morphological features, and it can also be regulated in a caspase-independent manner. Therefore, necroptosis can be understood initially as a combination of necrosis and apoptosis. The mechanism of its regulation, induction and inhibition is complicated, and involves a range of molecular expression and regulation. According to the recent literature, necroptosis takes place in the physiological regulatory processes of an organism and is involved in the occurrence, development and prognosis of a variety of diseases that have a necrosis phenotype, including neurodegenerative diseases, ischemic disease, hemorrhagic disease, inflammation and viral infectious diseases. In the
\end{abstract}

Correspondence to: Dr Gang Chen or Dr Qing Sun, Department of Neurosurgery, The First Affiliated Hospital of Soochow University, 188 Shizi Street, Suzhou, Jiangsu 215006, P.R. China

E-mail: nju_neurosurgery@163.com

E-mail: qsun@suda.edu.cn

${ }^{*}$ Contributed equally

Abbreviations: PCD, programmed cell death; DR, death receptors; TNF $\alpha$, tumor necrosis factor- $\alpha$; TRAIL, TNF-related apoptosis-inducing ligand; caspases, cysteine aspartyl proteases; PI, propidium iodide; Nec-1, necroptosis-specific inhibitor-1; RIP, receptor interacting protein; TNFR1, tumor necrosis factor receptor 1 ; ROS, reactive oxygen species; TRADD, TNF $\alpha$ receptor-associated death domain; TRAF, TNFR-associated factor; cIAP, cellular inhibitor of apoptosis; IKK, IкB kinase; NEMO, nuclear factor- $\kappa \mathrm{B}$ essential modulator; NF- $\mathrm{BB}$, nuclear factor- $\kappa \mathrm{B}$; MAPK, mitogen-activated protein kinase; FADD, Fas-associated protein with a death domain; MLKL, mixed lineage kinase domain-like protein; NMDA, N-methyl-D-aspartic acid

Key words: necroptosis, apoptosis, necrosis, cell death, inflammation, tumor necrosis factor $\alpha$-tumor necrosis factor receptor 1 , stroke present review, the features, molecular mechanism and identification of necroptosis under pathological conditions are discussed, with particular emphasis on its association with stroke.

\section{Contents}

1. Introduction

2. Cell death

3. Mechanisms of necroptosis and the TNFa-TNFR1-related signaling pathway

4. Necroptosis in stroke

5. Necroptosis in other diseases

6. Conclusions

\section{Introduction}

Cell death is an essential process in the physiology, pathology, growth, development, senility and death of an organism. There are two major modes of cell death: Apoptosis and necrosis, whose molecular mechanisms have been extensively studied (1). In the last few years, other forms of cell death have been identified and reported, including autophagy and necroptosis (2). Necroptosis is another form of cell death which was recently discovered; it not only has some similar phenotypes with necrosis, but can also be regulated by a series of exact mechanisms. It can be activated by stimulation with ligands of death receptors (DRs), such as TNF- $\alpha$ (3). However, the few studies on necroptosis in cerebrovascular diseases, such as stroke, are insufficient to form a comprehensive understanding of the topic. The present review mainly expounds the features, molecular mechanism [focusing on the tumor necrosis factor $\alpha$-tumor necrosis factor receptor 1 (TNF $\alpha$-TNFR 1 ) signaling pathway] and identification of necroptosis, and the latest study of this type of cell death in stroke.

\section{Cell death}

Cell death is an irreversible biological phenomenon and a termination of life. The death of nerve cells has different forms and features. Cell death was previously divided into necrosis and apoptosis. Necrosis can be recognized early and defined through pathomorphic characteristics, which occur when cells 
or the body is faced with enormous external pressure, and is a passive process. Meanwhile, apoptosis is regulated cell death, also known as programmed cell death (PCD) (4). A recent study showed, however, that necrosis can also be regulated in a programmed manner (3).

Cells undergo a type of passive death when organisms face up to enormous external pressure, and this is defined as necrosis. Necrosis was previously defined by its pathomorphic characteristics. Several causes, including physiological trauma, can lead to a change of internal environmental osmotic pressure, destroying the balance of an organism's internal environmental homeostasis and leading to necrosis (1). Furthermore, following necrosis, a series of reactions occur, including loss of cell membrane integrity, cell membrane swelling (due to absorption of moisture) and mitochondrial dysfunction (5). Due to the rupture of the cell membrane, a large number of intracellular elements leak out, which can cause and aggravate inflammation of the surrounding tissues (6).

Apoptosis is a natural phenomenon of cell death regulated by genes that occurs when organisms are affected by environmental stimulation (7). Damaged cells or cells without functions can be induced by protease (caspase) and then start the self-elimination procedure. As apoptosis occurs, a cell undergoes shrinkage, an increase in density, chromatin condensation, cracking of the nucleus and the formation of an apoptotic body (via cell membrane invagination). Due to the formation of the apoptotic body, the inflammatory response is avoided after apoptosis (8). In the majority of cases, apoptosis may be triggered in two ways: i) Intrinsic stimulation through the mitochondrial signaling pathway; or ii) extrinsic stimulation through cell surface DRs, including TNF $\alpha$, TNF-related apoptosis-inducing ligand (TRAIL) receptors and Fas (CD95/APO1). In either case, activation of cysteine aspartyl proteases (caspases) is necessary, and is known as the caspase-dependent manner (9). However, one previous study showed that apoptosis can occur even after caspases are inhibited. This result suggests that PCD can also occur in a caspase-independent manner (10).

Macroautophagy (referred to herein as autophagy) involves the engulfment of cytoplasmic material and intracellular organelles via a self-cannibalization mechanism, leading to the formation of autophagosomes. This is followed by fusion with lysosomes to form an autolysosome, where the encapsulated material is degraded by specific acidic hydrolases (11). In this way, cell would survive in a nutrient- or growth factor-deficient environment for a longer period; and there are some autophagy vacuolations formed in the cytoplasm, but not chromatin condenses (12). Autophagy is observed in physiological and pathological processes, however, the pros and cons of its role have not been fully elucidated.

Necroptosis is a form of cell death similar to necrosis in terms of morphological features (such as early destruction of membrane integrity, and cell and intracellular organelle swelling), but it can be regulated in a caspase-independent manner, that is to say that necroptosis can be triggered by a combination of death ligands and DRs under the inhibition of caspase (13). Additionally, necroptosis can be blocked by a small specific molecular compound known as necroptosis-specific inhibitor-1 (Nec-1), but not by any of the specific inhibitors of apoptosis or autophagy. At the same time, Nec-1 is unable to inhibit apoptosis and autophagy, and does not have any other significant effects on cells (14). As necroptosis occurs, the occurrence of autophagy can often be observed, however, necroptosis is not blocked after the application of inhibitors of autophagy, suggesting that autophagy is downstream of the necroptosis pathways (3). The most evident difference between necroptosis and apoptosis is the local inflammation caused by the release of the cell contents. The inflammation in necroptosis mainly presents as a large amount of inflammatory cell invasion and activation (15). Different forms and features of cell death, including certain connections and distinctions between these different forms are revealed in Fig. 1.

Distinguishing features of necroptosis. Although it has similar morphological characteristics, necroptosis is different from traditional necrosis, which is regulated by a series of signal transduction pathways and is a process of positive consumption of energy. Necroptosis ensures that cells are not completely passive when faced with external harm, in order to reduce damage to the cells via regulating a series of signals $(1,2)$. Necroptosis has typical morphological features of necrosis (organelles swelling, collapse, loss of cell membrane integrity and deficiency of nuclear chromatin), and the process can be identified through a light/electron microscope with propidium iodide (PI) staining (3). Due to the release of cell inclusions, necroptosis causes local inflammation, and infiltration and activation of inflammatory cells (15). Necroptosis also has the following characteristics: Cell death with necrotic characterization is more beneficial to maintain embryonic development and homeostasis during the adult period; necrotic cell death can be induced by a combination of ligands with their specific membrane receptors; necroptosis can be regulated via genetics, pharmacology and other factors; and inactivation of caspase can translate apoptosis into complete necrosis or a specific cell death with mixed characteristics of apoptosis and necrosis (3).

Identification of necroptosis. The basic identification of necroptosis can be divided into morphological and biochemical methods. Morphological methods include: Electron microscopy judgment and PI staining (16). Biochemical methods include: Use of necroptosis-specific inhibitor-1 (Nec-1), receptor interacting protein 1 (RIP1)-knockout and receptor interacting protein 3 (RIP3)-knockout. In addition, the formation of compounds (RIP1-RIP3), known as necrosomes, and the phosphorylation of RIP1 and RIP3 can serve as complementary judgment points $(17,18)$.

\section{Mechanisms of necroptosis and the TNF $\alpha$-TNFR1-related signaling pathway}

Following TNF signaling or other harmful stimulation that acts on corresponding cell death receptors, successive changes in mitochondria and the different forms of cell death occur (19). The mitochondrial outer membrane permeability is changed which leads to the release of the proteins in the mitochondrial intermembrane space, including cytochrome $c$. The release of cytochrome $c$ could activate apoptosis via mitochondrial pathways. Additionally, the release of apoptosis-inducing factor and endonuclease $\mathrm{G}$ is the start of caspase-independent apoptosis. Furthermore, changes in mitochondrial inner membrane 


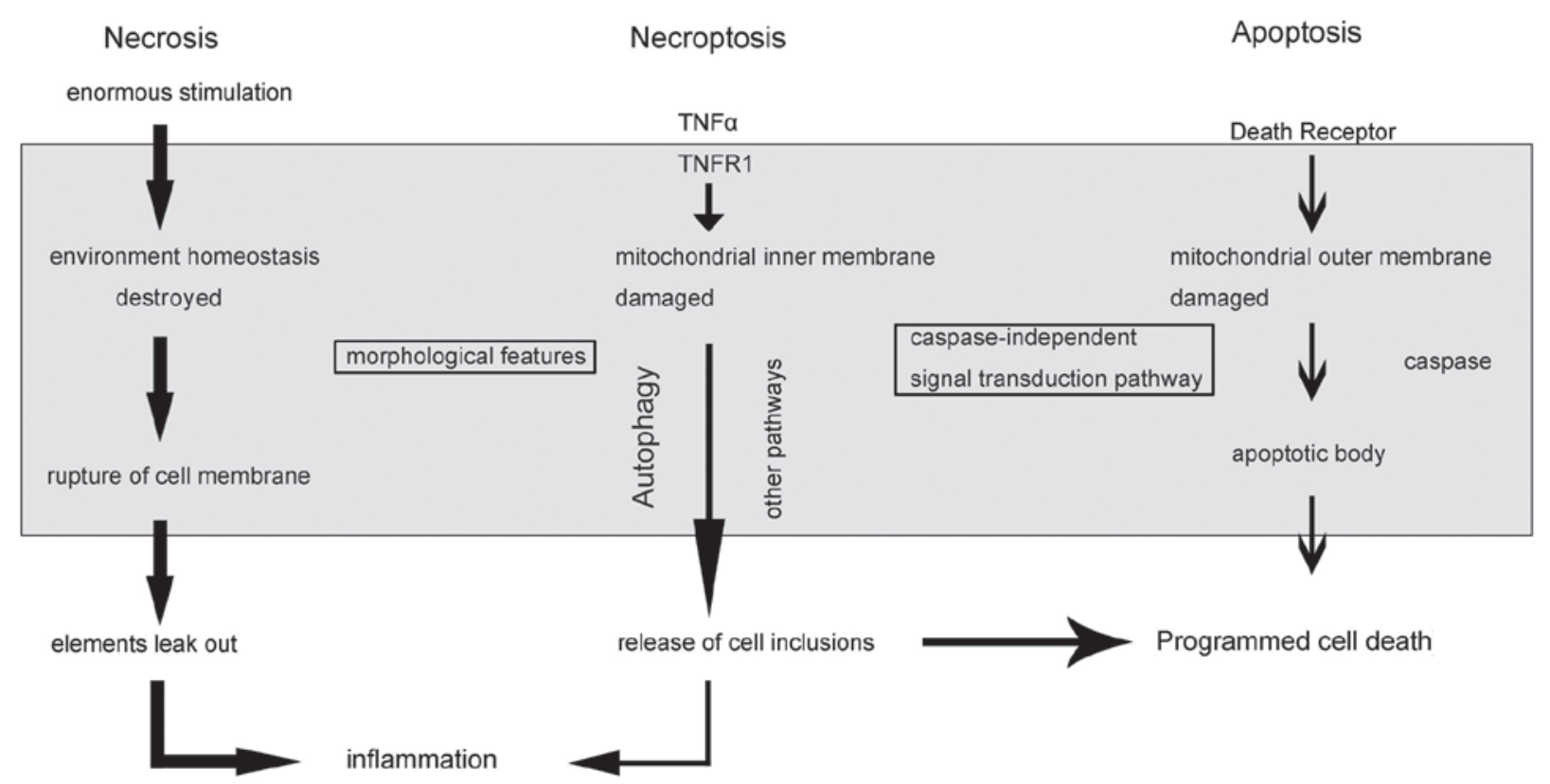

Figure 1. Different forms and features of cell death, including certain connections and distinctions between these different forms. TNFa, tumor necrosis factor- $\alpha$; TNFR1, TNF receptor 1 .

permeability induce a rapid decline in mitochondrial membrane potential, destroying respiratory chain electron transport (4). As a result, adenosine triphosphate synthesis and cell membranes are damaged, with necrosis-like cell death occurring (20). In short, damage of the mitochondrial outer membrane leads to apoptosis and damage of the mitochondrial inner membrane leads to necroptosis. Therefore, compared with apoptosis, necroptosis does not involve the key regulators of apoptosis factors (caspase) and does not involve the release of mitochondrial cytochrome $c$.

According to previous studies, there are several mechanisms of necroptosis, including the TNF $\alpha$-TNFR1-related signaling pathway, TRAIL and factors associated with the apoptosis ligand signaling pathway, the RIP3-mitochondrial-reactive oxygen species (ROS) metabolic pathway and the zVAD-mediated PKC-mitogen-activated protein kinase (MAPK)-AP-1-related signaling pathway (21-23). Among these mechanisms, the signaling cascade downstream of $\mathrm{TNF} \alpha$ leading to necroptosis has been deeply studied (24-28). In brief, TNFR1 exists in the cell membrane and its subunits can spontaneously trimerize at the plasma membrane (24). As ligands bind, the trimer conformation of these receptors would be changed, allowing their cytosolic tails to recruit multiple proteins, and then a complex (complex I) would develop near the cell membrane, which can include $\mathrm{TNF} \alpha$ receptor-associated death domain (TRADD), RIP1 kinase, TNFR-associated factor 2 (TRAF2), TRAF5, cellular inhibitor of apoptosis 1 (cIAP1) and cIAP2. Ubiquitylation of RIP1 by cIAP1 and cIAP2 stabilized complex I, and then combined with transforming growth factor $\beta$-activated kinase 1-binding protein 2 , I $\kappa$ B kinase (IKK) and nuclear factor- $\kappa \mathrm{B}$ essential modulator (NEMO) regulatory subunit (25). Afterwards, IKK $\alpha$ and IKK $\beta$ are activated and thus IKB can be phosphorylated, leading to nuclear factor- $\kappa \mathrm{B}(\mathrm{NF}-\kappa \mathrm{B})$ release and MAPK activation. The result of this NF- $\kappa B$ signaling pathway is the promotion of cell survival. RIP1 polyubiquitylation not only influences NF- $\kappa \mathrm{B}$ activation, but also affects the transition from complex I to II (26). Deubiquitylated RIP1 cooperates with its cognate kinase RIP3 for recruitment to another complex (complex II), which includes TRADD, FAS-associated protein with a death domain (FADD) and caspase-8. In this complex, RIP1 and RIP3 are inhibited by caspase- 8 and proteolytic cleavage is induced; therefore, the pro-apoptotic caspase activation cascade starts. By contrast, when caspase- 8 is inactivated, deficient or deleted, complex II does not initiate the apoptotic program and the TNF $\alpha$-mediated necroptotic pathway is used $(27,28)$. As a consequence, caspase- 8 and the interaction between RIP1 and RIP3 expression could be recognized as the diverging point of apoptosis and necroptosis (29). Nec-1 could halt the necroptosis signaling pathways by inhibiting RIPI and RIP3 kinase activity $(14,15)$. In addition, the formation of FADD-RIP1-RIP3-NEMO complex has an essential role on TNFa-mediated necroptotic pathways (30). A previous study identified the influence of the interaction of mixed lineage kinase domain-like protein (MLKL) and RIP3 (28). Later studies further supported the hypothesis that MLKL is indeed required for TNF $\alpha$-induced necroptosis, as key residues of MLKL mutants cannot be phosphorylated, which prevents the activation of the necrosome (31). A controversial point is whether the internal flow of $\mathrm{Ca}^{2+}$ or $\mathrm{Na}^{+}$ions is caused by MLKL-induced increased membrane permeability or by the formation of non-specific membrane channels. A summary of the TNF $\alpha$-TNFR1-related signaling pathway of cell survival, apoptosis and necroptosis is presented in Fig. 2.

\section{Necroptosis in stroke}

Stroke is a devastating illness second only to cardiac ischemia as a cause of mortality worldwide (32). Stroke used to occur mostly in the elderly, but in recent years patient age and age at mortality appears to have decreased, garnering attention to the problem. Cerebral vasospasm, a cerebral blood circulation 


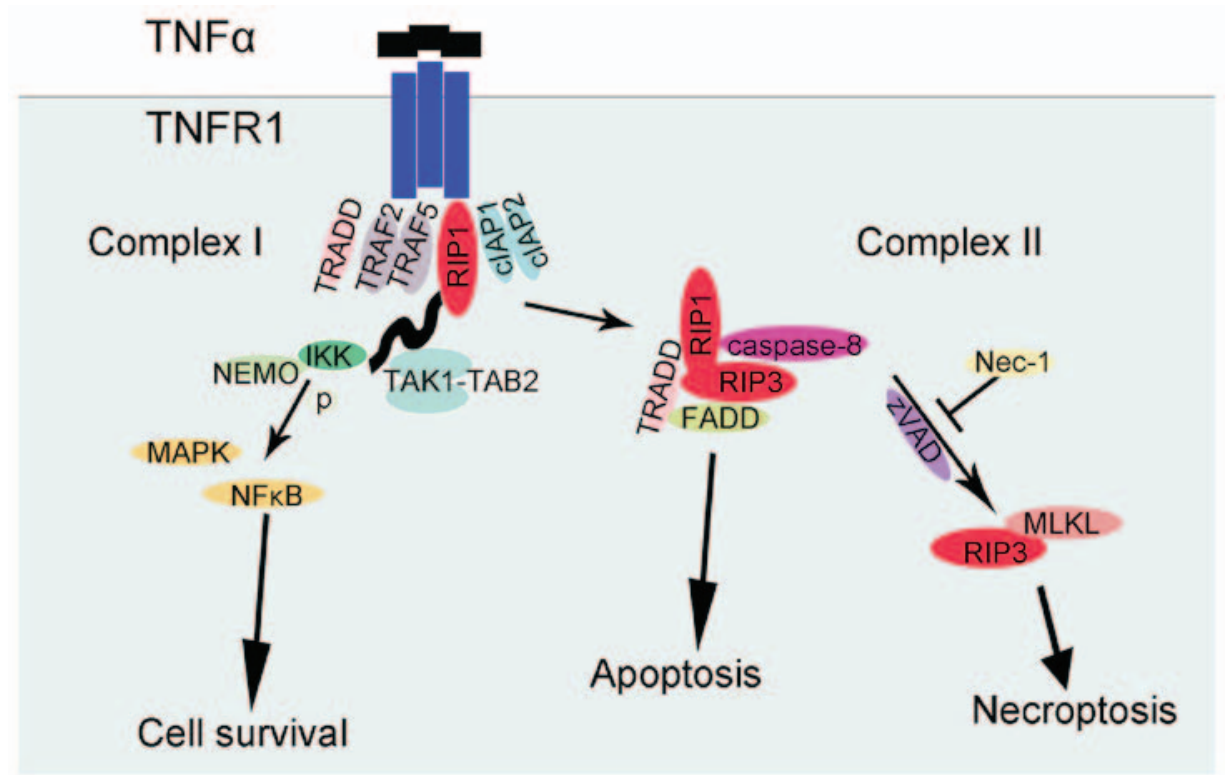

Figure 2. TNF $\alpha$-TNFR1-related signaling pathway of cell survival, apoptosis and necroptosis. TNF $\alpha$, tumor necrosis factor- $\alpha$; TNFR1, TNF receptor 1; Nec-1, necroptosis specific inhibitor-1; RIP1, receptor interacting protein; TRADD, TNF $\alpha$ receptor-associated death domain; TRAF, TNFR-associated factor; cIAP,

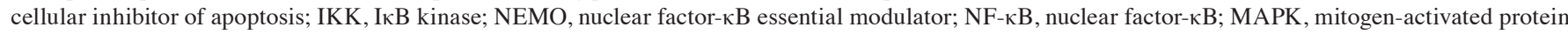
kinase; FADD, Fas-associated protein with a death domain; MLKL, mixed lineage kinase domain-like protein; TAK, transforming growth factor $\beta$-activated kinase 1; TAB2, TGF- $\beta$ activated kinase 1/MAP3K7 binding protein 2.

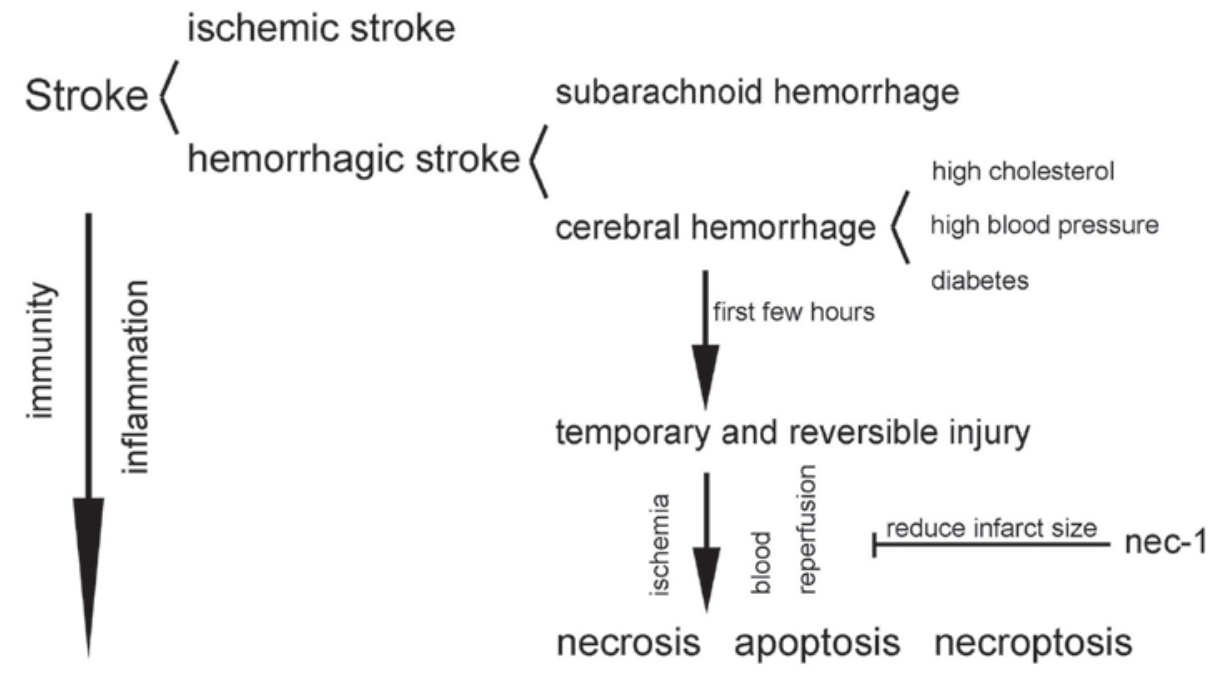

Figure 3. Association between three main forms of cell death and stroke. Nec-1, necroptosis specific inhibitor-1.

obstacle triggered by a sudden blockage or rupture of the cerebral vessels is the main cause of stroke. On this basis, brain tissues are damaged, and cranial nerve functions are partially or full disordered. Following the occurrence of stroke, immunity is a key element of the pathobiology, while the immune system participates in the brain damage, exerting a powerful immunosuppressive effect (33). Inflammatory signaling runs throughout all stages, from early damaging events triggered by arterial occlusion, to the late post-ischemic tissue repair (34).

Stroke is divided into two clinical categories: Ischemic stroke and hemorrhagic stroke. Ischemic stroke refers to the local brain tissues, including neural cells, glial cells and vasculature, becoming necrotic due to a lack of blood supply. The fundamental reason for this is that the blood system of the brain does not receive timely and sufficient collateral circulation after external and intracranial artery stenosis or with occluding lesions, thus decreasing the oxygen and energy supply, which the surrounding brain tissue metabolism requires. Hemorrhagic stroke refers to cerebral hemorrhage causing compression and necrosis of the brain tissue. This type of stroke is also known as a hemorrhagic cerebrovascular accident, and is generally divided into two categories: Cerebral hemorrhage and subarachnoid hemorrhage (33).

Cerebral hemorrhage is defined as a hemorrhage caused by non-traumatic brain parenchyma vascular rupture whose origin is mainly associated with a cerebrovascular lesion, such as caused by high cholesterol, diabetes and high blood pressure $(34,35)$. During the first few hours after a cerebral 
hemorrhage occurs, injury of the neurons in the ischemic penumbra and infarcted surrounding areas is temporary and reversible; apoptosis or necrosis is only an end result if no intervention occurs (36). Ischemic death of neurons exhibits mixed characteristics of apoptosis and necrosis in terms of biochemistry and ultrastructure (36). Ischemic cell damage is common in the clinic and can be divided into two stages, namely the reversible damage (can be repaired after injury if blood flow is reinstated) and irreversible damage (due to continuous ischemia, hypoxia and lack of basic metabolites). Cells mainly die by a necrosis-like process via dysfunction of the mitochondria in the irreversible lesion, but not all cell death is triggered by ischemia. Blood reperfusion, including some infiltration of neutrophils, cytokines and ROS, etc., can exacerbate tissue damage (36). At present, studies on ischemia/reperfusion in vivo are performed on animal models by blocking one side of the terminal artery of a certain organ or tissue (36). Furthermore, Nec-1 is the first found molecule that has been found to significantly reduce infarct size in the rat model of middle cerebral artery occlusion (3). The similar effects of Nec-1 can also be found in the study of ischemia-reperfusion damage in myocardial, retinal and kidney tissues (37-39). In addition, intracerebroventricular injection of Nec-1 can significantly reduce the infarction area in the middle cerebral artery occlusion model (3), and the protective effect of 7-Cl-O-Nec-1 injection can be detected $6 \mathrm{~h}$ after injury. However, the effect of zVAD-fmk (a caspase inhibitor) injection is extremely small at this point. That is to say the nerve protection effect of 7-Cl-O-Nec-1 can expand the therapeutic time window, and reveals the backwardness of necroptosis in cerebral ischemic injury (40). Furthermore, inflammatory factors increase significantly after cerebral hemorrhage, as detected via the immunohistochemical method. Activation of microglia can be used as another indicator to determine the occurrence and development of neuroinflammation (32). Microglia cells are inherent immune cells in the brain, maintaining a static phenotype in the normal state. As brain injury occurs, microglia cells are activated and migrate to the damaged regions prior to a response by any other cells in the body. At the same time, the quiescent branching shape of the microglia cells translates to an operative amoeba-like shape. Microglia cells swallow pathogens, apoptotic cells and cellular debris through proliferation, although their activation and concrete mechanisms remain unclear $(32,36)$. In Fig. 3, the associations between the three main forms of cell death and stroke are abstractly exhibited.

\section{Necroptosis in other diseases}

$N$-methyl-D-aspartic acid (NMDA) receptor-associated diseases. Glutamic acid is an important excitatory neurotransmitter in the central nervous system, and the activation of its receptor plays a significant role in physiological processes. Meanwhile, glutamic acid can also produce a neurotoxic effect via the excessive activation of NMDA receptor (41). A growing body of research has suggested that in numerous acute or chronic neurological diseases, including Huntington's disease, Parkinson's disease and Alzheimer's disease, this excitatory neurotoxicity is one of the main mechanisms of neuron death $(42,43)$. It has been proved that Nec-1 can suppress the decrease in cell vitality and the release of lactate dehydrogenase, and decrease the number of living cells induced by NMDA receptor (44). The results indicate that necroptosis is involved in NMDA-induced cellular damage.

Inflammatory diseases. Studies have shown the vital role of caspase- 8 in the regulation of necroptosis in intestinal epithelial cells and terminal ileum cells (45). Spontaneous formation of inflammatory damage can be observed in caspase-8-defective mice, and these mice are highly susceptible to colonitis (45). In patients with Crohn's disease (which exhibits characteristic necrosis of terminal ileum epithelial cells), as expression of RIP3 of intestinal epithelium goblet cells increases, the necroptosis of terminal ileum cells is enhanced, and this can be blocked by inhibitors of necroptosis, suggesting that RIP3-mediated necroptosis may take part in the genesis and development of inflammatory diseases (45).

Cancer. Necroptosis serves an indispensable role in the pathogenesis of cancer, as this process usually fails during tumorigenesis and tumor development (46). For instance, cells in chronic lymphocytic leukemia cannot undergo necroptosis when stimulated by TNF $\alpha$ and zVAD, and the key component of necroptosis, RIP3 is markedly downregulated (47). Besides, in non-Hodgkin lymphoma, single nucleotide polymorphisms in the RIP3 gene can be detected and are correlated with an increased risk of morbidity, indicating that genetic variations in the RIP3 gene may contribute to this cancer type (48). In addition, more and more compounds and anticancer agents have been discovered to induce necroptosis of cancer cells. Shikonin, a small molecule, was the first compound discovered to induce necroptosis in cancer (49) and a number of studies proved that shikonin and its analogs were highly antineoplastic without drug resistance (50-52).

Other diseases. In the mouse myocardial ischemia model, injecting Nec-1 after reperfusion can markedly shrink the volume of focal necrosis overall and in vitro (53). Additionally, in the mouse permanent ligation of coronary artery model, Nec-1 can significantly shrink the volume of necrosis, inhibit myocardial tissue fibrosis after necrosis and improve heart function. In the acute pancreatitis model, RIP3-knockout mice exhibit less necrosis of pancreatic cells, leakage of pancreatic enzyme and inflammation of pancreatic tissue $(17,28)$. Another study has reported that the PCD of capillary endothelial cells is composed of classic apoptosis and necroptosis. The classic apoptosis occurs from the original stage and throughout the whole growing phase, while necroptosis takes place only after appearance of capillary lumina and filtration barriers (37). Thus, we speculate that its significance lies in the fact that the collapsed contents of necroptosis cells can be taken away by the blood flow, preventing inflammation. Increasing numbers of studies are focusing on this aspect at present.

\section{Conclusions}

With the rapid progress of science and technology, a growing number of studies have shown that, other than the classic necrosis and apoptosis, cell death consists of another form, known as necroptosis. The occurrence and progression of 
necroptosis is based on specific signaling pathways (such as TNF- $\alpha$-TNFR1-related signaling pathway) and regulated by specific biological molecules (including RIP1, RIP3 and caspase-8). At the same time as the occurrence of necroptosis, inflammation occurs due to release of cellular inclusions causing infiltration and activation of inflammatory cells. Thus far, the specificity of a terminal target, which is similar to DNA fragmentation when apoptosis occurs, has not been found in the process of necroptosis. Currently, the basic judgment of necroptosis can be divided into the morphological method (electron microscopy and PI staining) and biochemical methods (Nec-1, RIP1-knockout or RIP3-knockout). The formation of necrosomes and the phosphorylation of RIP1 and RIP3 can serve as a complementary judgment indicator.

In clinical diseases, necroptosis is hypothesized to be associated with autophagy, apoptosis, necrosis and the inflammation of cells, providing a great possibility for future clinical combination drug therapy. These studies not only improve scientific literacy and technology, but also enrich the research of pathological mechanism in stroke and other diseases. More importantly, it may provide a knowledge base for clinical studies as well. In addition, due to the multiple signal regulatory pathways involved in necroptosis, the mechanism of necroptosis is extremely complicated and requires further study. Moreover, further research is also required to investigate the reasons for the inflammation caused by necroptosis and the varying functions of associated cells, including the activation and function of microglia cells.

\section{References}

1. Su Z, Yang Z, Xu Y, Chen Y and Yu Q: Apoptosis, autophagy, necroptosis, and cancer metastasis. Mol Cancer 14: 48, 2015.

2. Su Z, Yang Z, Xu Y, Chen Y and Yu Q: MicroRNAs in apoptosis, autophagy and necroptosis. Oncotarget 6: 8474-8490, 2015.

3. Degterev A, Huang Z, Boyce M, Li Y, Jagtap P, Mizushima N, Cuny GD, Mitchison TJ, Moskowitz MA and Yuan J: Chemical inhibitor of nonapoptotic cell death with therapeutic potential for ischemic brain injury. Nat Chem Biol 1: 112-119, 2005.

4. Nikoletopoulou V, Markaki M, Palikaras K and Tavernarakis N Crosstalk between apoptosis, necrosis and autophagy. Biochim Biophys Acta 1833: 3448-3459, 2013.

5. Schweichel JU and Merker HJ: The morphology of various types of cell death in prenatal tissues. Teratology 7: 253-266, 1973.

6. Leist $\mathrm{M}$ and Jäättelä M: Four deaths and a funeral: From caspases to alternative mechanisms. Nat Rev Mol Cell Biol 2: 589-598, 2001.

7. Yuksel S, Tosun YB, Cahill J and Solaroglu I: Early brain injury following aneurysmal subarachnoid hemorrhage: Emphasis on cellular apoptosis. Turk Neurosurg 22: 529-533, 2012.

8. Green DR: Apoptotic pathways: Ten minutes to dead. Cell 121: 671-674, 2005.

9. Adams JM: Ways of dying: Multiple pathways to apoptosis. Genes Dev 17: 2481-2495, 2003.

10. Kroemer G, Galluzzi L and Brenner C: Mitochondrial membrane permeabilization in cell death. Physiol Rev 87: 99-163, 2007.

11. Lévy J and Romagnolo B: Autophagy, microbiota and intestinal oncogenesis. Oncotarget 6: 34067-34068, 2015.

12. Ding WX, Ni HM, Gao W, Hou YF, Melan MA, Chen X, Stolz DB, Shao ZM and Yin XM: Differential effects of endoplasmic reticulum stress-induced autophagy on cell survival. J Biol Chem 282: 4702-4710, 2007.

13. Galluzzi L and Kroemer G: Necroptosis: A specialized pathway of programmed necrosis. Cell 135: 1161-1163, 2008.

14. Degterev A, Hitomi J, Germscheid M, Ch'en IL, Korkina O, Teng X, Abbott D, Cuny GD, Yuan C, Wagner G, et al: Identification of RIP1 kinase as a specific cellular target of necrostatins. Nat Chem Biol 4: 313-321, 2008.
15. You Z, Savitz SI, Yang J, Degterev A, Yuan J, Cuny GD, Moskowitz MA and Whalen MJ: Necrostatin-1 reduces histopathology and improves functional outcome after controlled cortical impact in mice. J Cereb Blood Flow Metab 28: 1564-1573, 2008.

16. Degterev A,Zhou W, Maki JL and Yuan J: Assays for necroptosis and activity of RIP kinases. Methods Enzymol 545: 1-33, 2014.

17. Zhang DW, Shao J, Lin J, Zhang N, Lu BJ, Lin SC, Dong MQ and Han J: RIP3, an energy metabolism regulator that switches TNF-induced cell death from apoptosis to necrosis. Science 325: 332-336, 2009.

18. Cho YS, Challa S, Moquin D, Genga R, Ray TD, Guildford M and Chan FK: Phosphorylation-driven assembly of the RIP1-RIP3 complex regulates programmed necrosis and virus-induced inflammation. Cell 137: 1112-1123, 2009.

19. Aggarwal BB: Signalling pathways of the TNF superfamily: A double-edged sword. Nat Rev Immunol 3: 745-756, 2003.

20. Orrenius S: Reactive oxygen species in mitochondria-mediated cell death. Drug Metab Rev 39: 443-455, 2007.

21. Jin Z and El-Deiry WS: Distinct signaling pathways in TRAIL-versus tumor necrosis factor-induced apoptosis. Mol Cell Biol 26: 8136-8148, 2006.

22. Vanden Berghe T, Declercq W and Vandenabeele P: NADPH oxidases: New players in TNF-induced necrotic cell death. Mol Cell 26: 769-771, 2007.

23. Wu YT, Tan HL, Huang Q, Sun XJ, Zhu X and Shen HM: zVAD-induced necroptosis in L929 cells depends on autocrine production of TNF $\alpha$ mediated by the PKC-MAPKs-AP-1 pathway. Cell Death Differ 18: 26-37, 2011.

24. Micheau O and Tschopp J: Induction of TNF receptor I-mediated apoptosis via two sequential signaling complexes. Cell 114: 181-190, 2003.

25. Ea CK, Deng L, Xia ZP, Pineda G and Chen ZJ: Activation of IKK by TNFalpha requires site-specific ubiquitination of RIP1 and polyubiquitin binding by NEMO. Mol Cell 22: 245-257, 2006.

26. Vandenabeele P, Galluzzi L, Vanden Berghe T and Kroemer G: Molecular mechanisms of necroptosis: An ordered cellular explosion. Nat Rev Mol Cell Biol 11: 700-714, 2010.

27. Feng S, Yang Y, Mei Y, Ma L, Zhu DE, Hoti N, Castanares M and Wu M: Cleavage of RIP3 inactivates its caspase-independent apoptosis pathway by removal of kinase domain. Cell Signal 19: 2056-2067, 2007.

28. He S, Wang L, Miao L, Wang T, Du F, Zhao L and Wang X: Receptor interacting protein kinase-3 determines cellular necrotic response to TNF-alpha. Cell 137: 1100-1111, 2009.

29. Deutsch M, Graffeo CS, Rokosh R, Pansari M, Ochi A, Levie EM, Van Heerden E, Tippens DM, Greco S, Barilla R, et al: Divergent effects of RIP1 or RIP3 blockade in murine models of acute liver injury. Cell Death Dis 6: e1759, 2015.

30. Irrinki KM, Mallilankaraman K, Thapa RJ, Chandramoorthy HC, Smith FJ, Jog NR, Gandhirajan RK, Kelsen SG, Houser SR, May MJ, et al: Requirement of FADD, NEMO, and BAX/BAK for aberrant mitochondrial function in tumor necrosis factor alpha-induced necrosis. Mol Cell Biol 31: 3745-3758, 2011.

31. Sun L, Wang H, Wang Z, He S, Chen S, Liao D, Wang L, Yan J, Liu W, Lei X, et al: Mixed lineage kinase domain-like protein mediates necrosis signaling downstream of RIP3 kinase. Cell 148: 213-227, 2012.

32. Iadecola $\mathrm{C}$ and Anrather J: The immunology of stroke: From mechanisms to translation. Nat Med 17: 796-808, 2011.

33. Engelhardt B and Sorokin L: The blood-brain and the blood-cerebrospinal fluid barriers: Function and dysfunction. Semin Immunopathol 31: 497-511, 2009.

34. Konsman JP, Drukarch B and Van Dam AM: (Peri)vascular production and action of pro-inflammatory cytokines in brain pathology. Clin Sci (Lond) 112: 1-25, 2007.

35. Jung KH, Yu KH, Kim YD, Park JM, Hong KS, Rha JH, Kwon SU, Bae HJ, Heo JH, Lee BC, et al: Antithrombotic management of patients with nonvalvular atrial fibrillation and ischemic stroke or transient ischemic attack: Executive summary of the Korean Clinical Practice Guidelines for Stroke. J Stroke 17: 210-215, 2015.

36. Wang J, Yang W, Xie H, Song Y, Li Y and Wang L: Ischemic stroke and repair: current trends in research and tissue engineering treatments. Regen Med Res 2: 3, 2014.

37. Oerlemans MI, Liu J, Arslan F, den Ouden K, van Middelaar BJ, Doevendans PA and Sluijter JP: Inhibition of RIP1-dependent necrosis prevents adverse cardiac remodeling after myocardial ischemia-reperfusion in vivo. Basic Res Cardiol 107: 270, 2012.

38. Rosenbaum DM, Degterev A, David J, Rosenbaum PS, Roth S, Grotta JC, Cuny GD, Yuan J and Savitz SI: Necroptosis, a novel form of caspase-independent cell death, contributes to neuronal damage in a retinal ischemia-reperfusion injury model. J Neurosci Res 88: 1569-1576, 2010. 
39. Linkermann A, Bräsen JH, Himmerkus N, Liu S, Huber TB, Kunzendorf U and Krautwald S: Rip1 (receptor-interacting protein kinase 1) mediates necroptosis and contributes to renal ischemia/reperfusion injury. Kidney Int 81: 751-761, 2012.

40. Zheng W, Degterev A, Hsu E, Yuan J and Yuan C: Structure-activity relationship study of a novel necroptosis inhibitor, necrostatin-7. Bioorg Med Chem Lett 18: 4932-4935, 2008.

41. Beal MF: Role of excitotoxicity in human neurological disease. Curr Opin Neurobiol 2: 657-662, 1992.

42. Tabrizi SJ, Cleeter MW, Xuereb J, Taanman JW, Cooper JM and Schapira AH: Biochemical abnormalities and excitotoxicity in Huntington's disease brain. Ann Neurol 45: 25-32, 1999.

43. Beal MF: Excitotoxicity and nitric oxide in Parkinson's disease pathogenesis. Ann Neurol 44 (Suppl 1): S110-S114, 1998.

44. Bonde C, Noraberg J, Noer H and Zimmer J: Ionotropic glutamate receptors and glutamate transporters are involved in necrotic neuronal cell death induced by oxygen-glucose deprivation of hippocampal slice cultures. Neuroscience 136: 779-794, 2005.

45. Günther C, Martini E, Wittkopf N, Amann K, Weigmann B Neumann H, Waldner MJ, Hedrick SM, Tenzer S, Neurath MF, et al: Caspase- 8 regulates TNF- $\alpha$-induced epithelial necroptosis and terminal ileitis. Nature 477: 335-339, 2011.

46. Fulda S: The mechanism of necroptosis in normal and cancer cells. Cancer Biol Ther 14: 999-1004, 2013
47. Liu P, Xu B, Shen W, Zhu H, Wu W, Fu Y, Chen H, Dong H, Zhu Y, Miao K, et al: Dysregulation of TNFo-induced necroptotic signaling in chronic lymphocytic leukemia: Suppression of CYLD gene by LEF1. Leukemia 26: 1293-1300, 2012.

48. Cerhan JR, Ansell SM, Fredericksen ZS, Kay NE, Liebow M, Call TG, Dogan A, Cunningham JM, Wang AH, Liu-Mares W, et al: Genetic variation in 1253 immune and inflammation genes and risk of non-Hodgkin lymphoma. Blood 110: 4455-4463, 2007.

49. Han W, Li L, Qiu S, Lu Q, Pan Q, Gu Y, Luo J and Hu X: Shikonin circumvents cancer drug resistance by induction of a necroptotic death. Mol Cancer Ther 6: 1641-1649, 2007.

50. Wada N, Kawano Y, Fujiwara S, Kikukawa Y, Okuno Y, Tasaki M, Ueda M, Ando Y, Yoshinaga K, Ri M, et al: Shikonin, dually functions as a proteasome inhibitor and a necroptosis inducer in multiple myeloma cells. Int J Oncol 46: 963-972, 2015.

51. Fu Z, Deng B, Liao Y, Shan L, Yin F, Wang Z, Zeng H, Zuo D, Hua Y and Cai Z: The anti-tumor effect of shikonin on osteosarcoma by inducing RIP1 and RIP3 dependent necroptosis. BMC Cancer 13: 580, 2013.

52. Xuan Y and Hu X: Naturally-occurring shikonin analogues - a class of necroptotic inducers that circumvent cancer drug resistance. Cancer Lett 274: 233-242, 2009.

53. Smith CC, Davidson SM, Lim SY, Simpkin JC, Hothersall JS and Yellon DM: Necrostatin: A potentially novel cardioprotective agent? Cardiovasc Drugs Ther 21: 227-233, 2007. 